\title{
Évolution des caractéristiques physico-chimiques et des paramètres de coagulation du lait de brebis collecté dans la région de Roquefort
}

\author{
O Pellegrini ${ }^{1}$, F Remeuf ${ }^{1}$, M Rivemale ${ }^{2}$ \\ ${ }^{1}$ Laboratoire de recherche de la chaire de technologie, INA Paris-Grignon/INRA, \\ 78850 Thiverval-Grignon; \\ 2 Société des caves de Roquefort, centre de recherche, 12250 Roquefort, France
}

(Reçu le 8 avril 1994; accepté le 22 août 1994)

\begin{abstract}
Résumé - Les caractéristiques physico-chimiques et l'aptitude à la coagulation par la présure du lait de brebis collecté dans la région de Roquefort ont été suivies au cours d'une période de lactation. Les résultats obtenus mettent en évidence une évolution nette de la composition globale du lait au cours de la période de production. L'étude de la répartition du calcium et du phosphore inorganique entre les phases soluble et colloïdale fait apparaître une augmentation de la fraction colloïdale. Concernant les caractéristiques micellaires, on note un accroissement du diamètre des micelles et une diminution de la minéralisation calcique. Le degré d'hydratation des micelles et les proportions relatives des caséines évoluent peu. Le suivi des paramètres de coagulation montre une augmentation nette du temps de prise, s'accompagnant d'une réduction de la vitesse de raffermissement. La fermeté du gel croît légèrement et l'aptitude à l'exsudation du lactosérum diminue.
\end{abstract}

lait de brebis / caractéristique physico-chimique / équilibre salin / micelle / coagulation présure

Summary - Evolution of physico-chemical characteristics and renneting properties of ewe's milk collected in the 'Roquefort area'. In the present work, we studied the composition of ewe's milk collected in the 'Roquefort area'. Results show real evolutions of milk composition throughout the lactation period. The study of the partition of calcium and inorganic phosphorus between the aqueous and colloidal phases shows that the concentration of colloidal components decreases during the lactation period. An increase of the mean diameter of casein micelles is observed, whereas the micelle mineralization decreases. The micelle solvatation and the proportions of $\alpha_{s 1}, \alpha_{s 2}, \beta$ and $\kappa$ caseins show little evolutions. Variations of the coagulation parameters throughout the lactation suggest that the rennet coagulation time rises while the gel firming rate becomes slower with advancing lactation time. The curd firmness increases weakly and the whey draining capacity of the curd decreases.

ewe's milk / physico-chemical characteristic / salt distribution / micelle / rennet coagulation 


\section{INTRODUCTION}

Plusieurs publications récentes (eg Ramos et Juarez, 1981 ; Assenat, 1985 ; Anifantakis, 1986 ; Nuñes et al, 1989) font le point des connaissances sur la composition et les propriétés physico-chimiques du lait de brebis. Elles s'appuient sur des données bibliographiques dans l'ensemble moins nombreuses que celles relatives au lait de vache. Les caractéristiques du lait ovin évoluent de façon significative au cours de la période de lactation. Toutefois, compte tenu de la saisonnalité du cycle de reproduction des brebis, il est difficile de distinguer les influences respectives du stade de lactation, de la saison et de l'alimentation (Ramos et Juarez, 1981).

Quels que soient la race et le mode d'élevage, la quasi-totalité des auteurs notent une augmentation nette et régulière de la teneur en protéines du lait au cours de la lactation (eg Assenat, 1985 ; Voutsinas et al, 1988 ; Muir et al, 1993). En ce qui concerne la teneur en matière grasse, les évolutions décrites peuvent être assez différentes selon les races ou les régions d'élevage (eg Assenat, 1985 ; Voutsinas et al, 1988 ; Lemoine, 1990; Muir et al, 1993). Assenat (1985) et Lemoine (1990) observent pour des brebis de race Lacaune et Manech élevées en France, une augmentation quasi linéaire du taux butyreux.

La teneur en lactose des laits de brebis tend à diminuer plus ou moins régulièrement au cours de la lactation (Assenat, 1985 ; Lemoine, 1990 ; Muir et al, 1993). Les concentrations en matières salines (Assenat, 1985; Polychroniadou et Vafopoulou, 1985 ; Voutsinas et al, 1988) et leur répartition entre les phases soluble et colloïdale (O'Connor et Fox, 1977 ; Holt et Jenness, 1984 ; Polychroniadou et Vafopoulou, 1986 ; Lemoine, 1990) ne montrent pas, dans lensemble, d'évolutions significatives.
En ce qui concerne les caractéristiques micellaires, les résultats publiés ne sont pas toujours comparables, du fait de l'utilisation de techniques d'analyse différentes. Ainsi en est-il de la taille des micelles, mesurée par microscopie électronique (Richardson et al, 1974 ; Saleem et al, 1986) ou par diffusion lumineuse (Lemoine, 1990). De même, les données concernant les proportions relatives des caséines obtenues par électrophorèse (Brochet, 1982 ; Juarez et al, 1984) ou par des techniques chromatographiques (Ono et al, 1989 ; Law et al, 1992 ; Muir et al, 1993) présentent une certaine hétérogénéité.

Les évolutions de la composition du lait de brebis, notamment en matière protéique, laissent présager des variations non négligeables de son comportement vis-à-vis de la présure. Les travaux relatifs à l'aptitude à la coagulation du lait de brebis se sont attachés généralement à faire la comparaison avec les laits d'autres espèces (Storry et al, 1983). D'autres études ont permis de mettre en évidence l'influence de la composition sur les caractéristiques lactodynamiques (Manfredini et al, 1987 ; Ubertalle et al, 1990 ; Duranti et Casoli, 1991 ; Manfredini et al, 1992). Seuls quelques articles (eg Ubertalle et al, 1990 ; Delacroix-Buchet et al, 1994) décrivent l'évolution des paramètres de coagulation (temps de prise, vitesse de raffermissement, fermeté du gel) au cours de la période de lactation.

L'objectif principal de cette étude est de préciser l'évolution des caractéristiques physico-chimiques (fractions azotées, caractéristiques micellaires, équilibres salins) et des paramètres de coagulation du lait de brebis collecté dans la région de Roquefort. Par ailleurs, les données recueillies complètent les connaissances actuelles relatives à certains aspects peu ou non étudiés de la physico-chimie du lait de brebis. 


\section{MATÉRIEL ET MÉTHODES}

\section{Dispositif expérimental. Échantillonnage et prélèvements}

Les caractéristiques physico-chimiques et les paramètres de coagulation du lait de brebis de la région de Roquefort ont été étudiés au cours des périodes de production de 1991 et 1992.

En 1991, le lait collecté par trois fromageries de la Société des caves de Roquefort a été analysé. Le lait mis en fabrication dans chaque fromagerie a été échantillonné de 15 à 20 fois entre les mois de janvier et juillet, ce qui correspond à un prélèvement par fromagerie tous les 10 à 12 jours. Sur ces échantillons, ont été déterminées les caractéristiques de composition globale, c'est-à-dire le taux de matière grasse, les concentrations des différentes fractions azotées, et celles du lactose, du calcium, du phosphore et du citrate. Les proportions relatives des caséines ont été évaluées sur le lait collecté par l'une des fromageries.

En 1992, les prélèvements ont été effectués sur le lait d'une seule fromagerie, la méthodologie d'échantillonnage et la fréquence des prélèvements étant identiques à celles de 1991. Ces laits ont fait l'objet d'une analyse comportant la caractérisation des équilibres salins (calcium et phosphore inorganique totaux et solubles), la mesure des caractéristiques micellaires (diamètre moyen, degré d'hydratation et minéralisation calcique) ainsi que la détermination des paramètres de coagulation.

Le protocole d'échantillonnage est le suivant: le mélange des laits de la traite du soir $(\mathrm{J}-1)$ et du matin $(\mathrm{J})$, conservé en ferme à la température de $9-10^{\circ} \mathrm{C}$, est collecté quotidiennement. À la fromagerie, le lait est stocké dans 3 ou 4 tanks de 25000 l entre 5 et $7^{\circ} \mathrm{C}$, et brassé 2 minutes toutes les heures. Avant la fabrication, au jour $\mathrm{J}+1$, le lait est soumis à un brassage continu pendant 15 minutes, puis dirigé vers les cuves de fabrication. C'est au cours de cette phase d'agitation qu'est effectué l'échantillonnage. Un litre de lait est prélevé dans un des tanks et conservé à $4-6^{\circ} \mathrm{C}$. La flore totale des laits, contrôlée avant chaque fabrication, est inférieure à $5 \cdot 10^{4} \mathrm{ufc} / \mathrm{ml}$. L'échantillon est acheminé à Roquefort à $\mathrm{J}+2$ où il est additionné d'azothydrate de sodium $(0,4 \mathrm{~g} / \mathrm{l})$ et de phénylméthylsulfonyl fluoride (PMSF) $(0,045 \mathrm{~g} / \mathrm{l})$. Envoyé par Chronopost, le lait est réceptionné à Grignon à $\mathrm{J}+3$.
Le début de la collecte se situe pendant la première quinzaine de décembre pour deux des trois fromageries, et pendant la deuxième quinzaine pour la troisième. Dans la présentation des résultats, le temps $t_{0}$ correspond au premier jour de la quinzaine d'ouverture de chaque fromagerie.

\section{Détermination des caractéristiques physico-chimiques}

Les analyses effectuées en 1991 par le laboratoire de la Société des caves de Roquefort, et en 1992 par le laboratoire de la chaire de technologie à Grignon, sont réalisées le jour mème de la réception de l'échantillon.

Le lait est réchauffé à $30^{\circ} \mathrm{C}$, mélangé par agitation lente puis ramené à $20^{\circ} \mathrm{C}$. Les échantillons sont conservés au minimum $1 \mathrm{~h}$ à cette température avant l'analyse. Toutes les déterminations sont effectuées en double et les résultats sont exprimés en $\mathrm{g} / \mathrm{l}$ de lait entier excepté les teneurs en calcium et phosphore inorganique mesurées en1992 qui sont exprimées en $\mathrm{g} / \mathrm{l}$ de lait écrémé.

\section{Fractionnement et dosage des matières azotées}

Le dosage de l'azote dans les différentes fractions est effectué par la méthode Kjeldahl. Sont ainsi déterminés: lazote total (NT), l'azote soluble à $\mathrm{pH} 4,6$ (NST) préparé selon la méthode de Rowland (1938), et l'azote soluble dans le TCA $12 \%$ ou azote non protéique (NPN). Les teneurs en azote des différentes fractions sont exprimées en équivalent de matière azotée selon les formules suivantes :

- protéines totales $=(\mathrm{NT}-\mathrm{NPN}) \times 6,38$;

- caséines $=(\mathrm{NT}-\mathrm{NST}) \times 6,38$;

- protéines solubles $=($ NST - NPN $) \times 6,38$.

La teneur en azote non protéique est exprimée en g d'azote/l. L'urée fait l'objet d'un dosage á l'aide d'un kit enzymatique Boehringer (Boehringer Mannheim France, Meylan, France).

\section{Matière grasse et lactose}

La teneur en matière grasse est déterminée à l'aide de la méthode acidobutyrométrique de Gerber (norme AFNOR NF V04-210). Le lactose est dosé par une méthode enzymatique (Boehringer). 


\section{Matières salines}

En 1991, les teneurs en calcium, phosphore et citrate sont déterminées sur lait entier. Le calcium est dosé par fluorimétrie à l'aide d'un calcimètre Corning Calcium Analyser 940 (Laboratoires Humeau, La Chapelle-sur-Erdre, France). La concentration en phosphore total est déterminée à l'aide d'une méthode colorimétrique basée sur la formation, en milieu acide, d'un complexe coloré de phosphomolybdate d'ammonium (norme FIL 42, 1967). La teneur en citrate total est appréciée par une méthode enzymatique (Boehringer). En 1992, les caractéristiques des équilibres salins sont déterminées sur lait écrémé par centrifugation à $1500 \mathrm{~g}$ pendant 20 minutes à $20^{\circ} \mathrm{C}$. La séparation des phases soluble et colloïdale est effectuée par ultracentrifugation à $80000 \mathrm{~g}$ pendant $1 \mathrm{~h}$ à $20^{\circ} \mathrm{C}$. Les concentrations de calcium et de phosphore inorganique sont déterminées dans le lait et le sérum selon les protocoles décrits par Remeuf et al (1989). Le calcium ionisé est dosé sur le lait par potentiométrie (Remeuf et al, 1989) à l'aide d'une électrode spécifique Metrohm E $301 \mathrm{Ca}$ (Roucaire, Velizy-Villacoublay, France).

\section{Caractéristiques micellaires}

Le diamètre moyen et le degré d'hydratation des micelles sont déterminés selon les méthodes décrites par Remeuf et al (1989). Les dimensions micellaires sont estimées à l'aide d'un appareil Coulter N4 (Coultronics France, Margency, France) dont le principe de mesure repose sur la détermination du coefficient de diffusion quasi élastique d'un rayonnement laser. Le degré d'hydratation des micelles correspond à la teneur en eau du culot d'ultracentrifugation $(80000 \mathrm{~g}$ pendant $1 \mathrm{~h}$ à $20^{\circ} \mathrm{C}$ ) obtenue par pesée avant et après dessiccation $16 \mathrm{~h}$ à $103^{\circ} \mathrm{C}$.

Les proportions relatives des caséines sont évaluées par chromatographie HPLC en phase inverse sur une colonne Beckman Ultrapore $\mathrm{C}_{3}$ (Beckman Instruments France, Gagny, France). La longueur d'onde de détection est de $220 \mathrm{~nm}$. Le pourcentage relatif de chacune des caséines $\alpha_{\mathrm{s} 1}, \alpha_{\mathrm{s} 2}, \beta$ et $\kappa$ est calculé par le rapport des surfaces des pics d'absorption. En l'absence de données bibliographiques sur les différences d'absorption des caséines ovines à $220 \mathrm{~nm}$, aucun coefficient correctif n'a été utilisé. Les conditions opératoires, proches de celles décrites par Jaubert et Martin (1992), font appel à un gradient de 40 à $60 \%$ entre le solvant $\mathrm{A}$ (eau milliQ $+0,1 \%$ acide trifluoroacétique) et le solvant B (acétonitrile $80 \%+0,1 \%$ acide trifluoroacétique) (fig 1). Le débit est fixé à $1,5 \mathrm{ml} / \mathrm{min}$.

L'échantillon de caséine est préparé par précipitation acide $(\mathrm{HCl} 1 \mathrm{~N})$ à pH 4,6 de $20 \mathrm{ml}$ de lait écrémé dilué au demi. Le précipité est séparé par centrifugation (2 $000 \mathrm{~g} / 10 \mathrm{~min}$ ) et lavé 3 fois à l'eau bidistillée, le pH étant maintenu à 4,6. La caséine isoélectrique est réduite à létat de poudre sèche par traitement à l'acétone. Pour l'analyse, $20 \mathrm{mg}$ de poudre de caséine sont solubilisés dans $1 \mathrm{ml}$ d'une solution d'uree $8 \mathrm{~mol} / \mathrm{l}+$ dithiothreitol (DDT) $0,2 \%$. La quantité de caséine injectée est d'environ $25 \mu \mathrm{g}$.

\section{Aptitude du lait à la coagulation par la présure}

Les paramètres caractérisant la cinétique de coagulation du lait sont déterminés à l'aide d'un Formagraph (Foss Electric France, Paris, France) (Zannoni et Annibaldi, 1981). Les mesures sont effectuées en double sur le lait entier maintenu au préalable pendant 30 minutes à la température d'emprésurage, fixée à $30^{\circ} \mathrm{C}$. La dose de présure est équivalente à $26,66 \mathrm{ml}$ de présure pour 100 I (Présure Gand Gassiot à 520 mg/l de chymosine, Laboratoire Granday Roger, Beaune, France). L'activité coagulante de la présure est contrôlée au début de chaque journée d'analyse sur un échantillon témoin préparé à partir d'une poudre de lait (Journal Officiel, 1981). Le temps de prise (Tp) est le temps séparant l'emprésurage du point d'écartement des branches de la courbe ( $\mathrm{min}$ ). La vitesse de raffermissement du gel (VRG), exprimée en $\mathrm{mm} / \mathrm{min}$, est calculée à partir du temps nécessaire pour obtenir un écartement de $30 \mathrm{~mm}$ entre les 2 tracés de l'enregistrement $\left(V R G=30 / K_{30}\right)$. La fermeté du gel Ar, (en $\mathrm{mm}$ ) correspond à l'écartement mesuré à 2 fois le temps de prise.

\section{Aptitude à l'égouttage}

La détermination de l'aptitude à l'égouttage s'inspire de la méthode décrite par Marshall (1982); $25 \mathrm{~g}$ de lait, contenus dans un bëcher de $50 \mathrm{ml}$, 


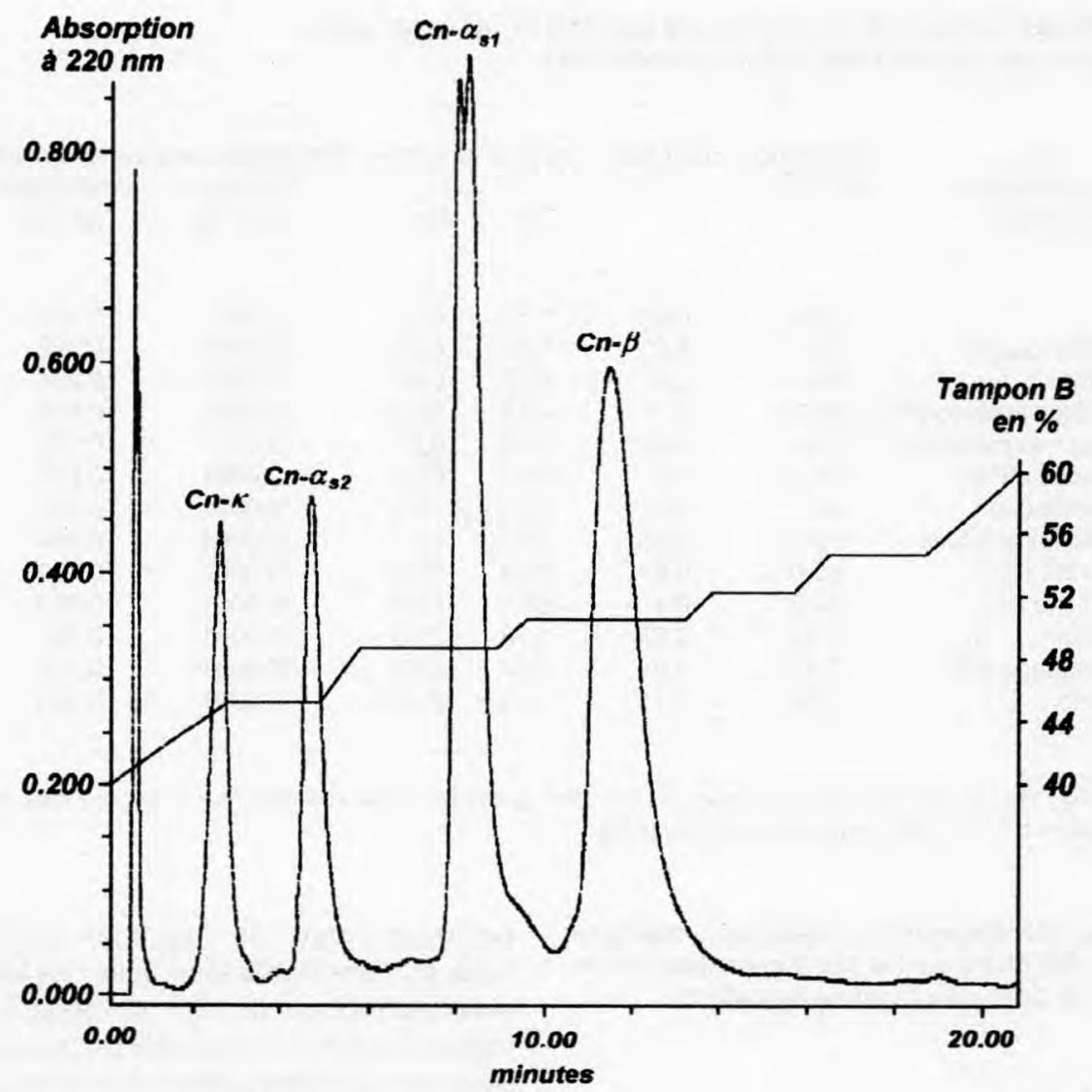

Fig 1. Séparation par HPLC en phase inverse des caséines ovines. RP-HPLC separation of ovine caseins.

sont mis au bain d'eau à $30^{\circ} \mathrm{C}$, maintenus à cette température pendant 30 minutes, puis emprésurés avec $125 \mu \mathrm{l}$ de présure Gand Gassiot diluée au $1 / 20^{\circ}$. Le gel obtenu après 60 minutes de coagulation est découpé en 12 cubes à l'aide d'un tranche-caillé de forme appropriée. Une heure après le découpage, on mesure la quantité de sérum qui s'écoule pendant 1 minute du bêcher incliné de $120^{\circ}$ par rapport à l'horizontale. Le gel est retenu dans le bêcher grâce à une grille placée à sa surface. La quantité de sérum recueilli est pesée. On exprime le résultat en pourcentage $(\mathrm{p} / \mathrm{p})$ de sérum exsudé par rapport à la quantité de lait emprésuré.

\section{Traitement statistique des données}

Les données de 1991 ont fait l'objet d'une analyse de variance visant à estimer les effets éventuels des facteurs "fromagerie" (3 niveaux) et "période" sur les variables mesurées. Le facteur "période" comporte 14 niveaux correspondant à 14 intervalles de 15 jours : chaque lait a été assigné à l'une des quinzaines en fonction de sa date de prélèvement par rapport à la date de référence $t_{0}$.

Le traitement statistique des données est effectué avec le logiciel SAS (SAS Institute, Inc, 
Tableau I. Composition moyenne du lait de brebis (en $\mathrm{g} / \mathrm{l}$ de lait entier). Mean composition of ewe's milk (in g/l whole milk).

\begin{tabular}{|c|c|c|c|c|c|c|}
\hline \multirow{2}{*}{$\begin{array}{c}g / \\
\text { (sauf indication } \\
\text { contraire) }\end{array}$} & \multirow{2}{*}{$\begin{array}{l}\text { Moyenne } \\
(\mathrm{n}=51)\end{array}$} & \multirow[t]{2}{*}{ Écart type } & \multicolumn{2}{|c|}{ Valeurs extrêmes } & \multirow{2}{*}{$\begin{array}{c}\text { Probabilités assoc } \\
\text { "période" } \\
(d l=12)\end{array}$} & \multirow{2}{*}{$\begin{array}{l}\text { ciées aux effets } \\
\text { "fromagerie" } \\
\quad(d l=2)\end{array}$} \\
\hline & & & Min & Max & & \\
\hline $\mathrm{pH}$ & 6,63 & 0,03 & 6,57 & 6,71 & 0,0001 & 0,140 \\
\hline Matière grasse & 74,0 & 8,8 & 59,0 & 88,5 & 0,0001 & 0,392 \\
\hline Lactose & 46,6 & 2,4 & 41,2 & 50,2 & 0,0001 & 0,031 \\
\hline Matières azotées (NT) & 56,3 & 4,4 & 48,4 & 62,6 & 0,0001 & 0,605 \\
\hline Azote non protéique & 0,44 & 0,04 & 0,36 & 0,51 & 0,0001 & 0,536 \\
\hline Protéines (Pro) & 53,5 & 4,5 & 45,1 & 60,1 & 0,0001 & 0,616 \\
\hline Caséine (Cnt) & 44,1 & 3,6 & 37,1 & 49,5 & 0,0001 & 0,669 \\
\hline Protéines solubles & 9,46 & 0,96 & 7,5 & 11,1 & 0,0001 & 0,162 \\
\hline Pro / NT (\%) & 95,0 & 0,8 & 93,3 & 96,2 & 0,0002 & 0,799 \\
\hline Cnt / Pro (\%) & 82,3 & 0,4 & 81,1 & 83,6 & 0,0001 & 0,050 \\
\hline Calcium & 1,89 & 0,07 & 1,78 & 2,08 & 0,0001 & 0,093 \\
\hline Phosphore total & 1,41 & 0,04 & 1,32 & 1,53 & 0,0001 & 0,016 \\
\hline Citrate & 1,93 & 0,11 & 1,75 & 2,18 & 0,0001 & 0,369 \\
\hline
\end{tabular}

Modèle : $Y i j k=\mu+\alpha i+\beta j+$ Eijk. $\mu=$ moyenne ; $\alpha i=$ effet "quinzaine" (effet "période") $1<i<14 ; \beta j=$ effet "fromagerien $1<j<3$; Eijk = erreur aléatoire résiduelle.

Cary, NC, Etats-Unis) à l'aide des procédures Proc MEANS pour les statistiques descriptives et Proc GLM pour l'analyse de variance.

\section{RÉSULTATS}

\section{Composition globale}

Le tableau I présente les valeurs moyennes, les valeurs extrêmes et les écart types des principales caractéristiques physicochimiques du lait collecté en 1991 par les 3 fromageries.

Les évolutions des principaux constituants du lait au cours de la période de production sont présentées figure 2. Le taux butyreux augmente de façon quasiment linéaire, passant de $60 \mathrm{~g} / \mathrm{l}$ à près de $90 \mathrm{~g} / \mathrm{l}$. Les courbes d'évolution des teneurs en protéines totales, caséines et protéines solubles sont très voisines : elles connaissent une croissance marquée durant les premiers mois, puis une stabilisation, voire une légère diminution à partir du $160^{\circ}$ jour environ. La teneur en azote non protéique diminue régulièrement, avec des valeurs comprises entre $0,49 \mathrm{~g} / \mathrm{l}$ en début de lactation et $0,37 \mathrm{~g} / \mathrm{l}$ en fin de période. L'urée suit une évolution semblable : c'est le constituant prédominant de l'azote non protéique, avec une teneur moyenne de 0,21 g/l d'azote, représentant $48 \%$ de cette fraction.

En ce qui concerne les proportions relatives des différents constituants de la matière azotée, l'étude révèle une augmentation du pourcentage des protéines par rapport à la matière azotée totale, qui passe de $93,5 \%$ à $96 \%$ entre le début et la fin de la période de production. En revanche, la proportion de caséine dans les protéines tend à diminuer très légèrement, de $82,9 \%$ à $82,1 \%$.

La concentration en lactose décroît régulièrement, passant de valeurs proches de $49 \mathrm{~g} / \mathrm{l}$ à environ $42 \mathrm{~g} / \mathrm{l}$ (fig 2). Les concentra- 


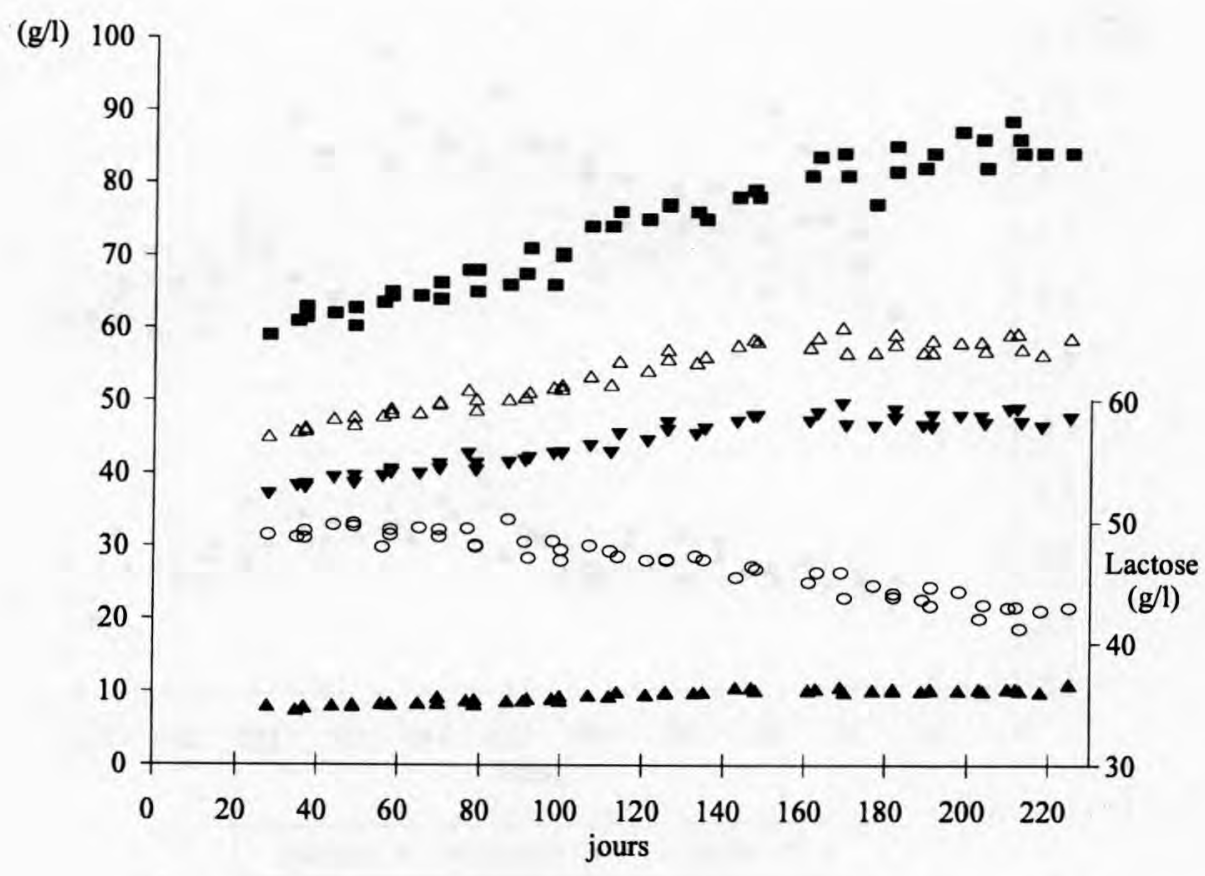

Fig 2. Évolution de la composition du lait de brebis : principaux constituants. Evolution of the composition of ewe's milk: main components.

tions des constituants salins : calcium, phosphore et citrate suivent des évolutions similaires (fig 3). Les valeurs obtenues en fin de lactation sont proches de celles rencontrées au début de la période de production. Les teneurs les plus élevées sont enregistrées entre le $120^{\circ}$ et le $160^{\mathrm{e}}$ jour de collecte. Quant au $\mathrm{pH}$, il diminue pendant les 100 premiers jours de collecte, passant de 6,70 à 6,62 puis se stabilise autour de cette valeur.

\section{Équilibres salins}

Les teneurs moyennes en calcium et phosphore inorganique totaux des laits analysés en 1992 sont égales respectivement à 2,10 et $0,96 \mathrm{~g} / \mathrm{l}$ de lait écrémé. Avec des concentrations moyennes de $1,63 \mathrm{~g} / \mathrm{l}$ et de $0,59 \mathrm{~g} / \mathrm{l}$, le calcium et le phosphore inorganique colloïdaux représentent respectivement $78 \%$ et $62 \%$ du calcium et du phosphore inorganique totaux. La concentration en calcium ionisé est de $0,13 \mathrm{~g} / \mathrm{l}$.

La figure 4 présente les variations des pourcentages de calcium et de phosphore inorganique solubles. On constate une diminution régulière et significative de la forme soluble de ces deux éléments. La proportion de calcium soluble passe de 24,5 à $20 \%$ du calcium total. La fraction soluble du phosphore inorganique, égale à $43 \%$ en début 


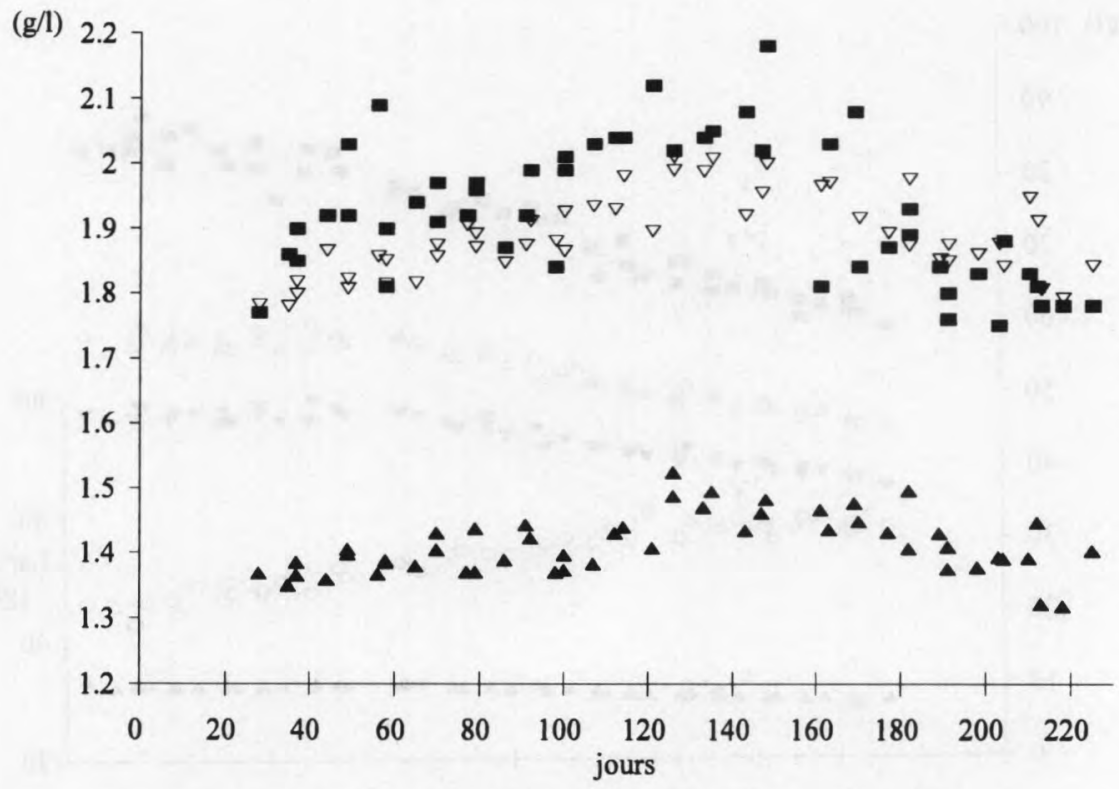

$$
\nabla \text { citrate } \quad \text { phosphore }=\text { calcium }
$$

Fig 3. Évolution de la composition du lait de brebis en matières salines.

Evolution of the salt concentrations in ewe's milk.

Tableau II. Caractéristiques micellaires et proportions relatives des caséines.

Micelle characteristics and casein proportions.

\begin{tabular}{|c|c|c|c|c|}
\hline \multirow{3}{*}{$\begin{array}{l}\text { Degré d'hydratation } \\
\text { DHM (g eau/g MS) }\end{array}$} & \multirow{3}{*}{$\begin{array}{c}\begin{array}{c}\text { Moyenne } \\
(\mathrm{n}=20)^{*}\end{array} \\
1,82\end{array}$} & \multirow{3}{*}{$\begin{array}{c}\text { Écart type } \\
0,04\end{array}$} & \multicolumn{2}{|c|}{ Valeurs extrêmes } \\
\hline & & & Min & \\
\hline & & & 1,71 & 1,93 \\
\hline $\begin{array}{l}\text { Diamètre moyen } \\
\text { DMM }(n m)\end{array}$ & 193 & 7,3 & 173 & 207 \\
\hline $\begin{array}{l}\text { Minéralisation calcique MIC } \\
\text { (mg Ca/g caséine) }\end{array}$ & 33,4 & 1,78 & 30,8 & 36,5 \\
\hline$\%$ caséine $\alpha_{\mathrm{s} 1}$ & 34,5 & 1,1 & 32,5 & 36,1 \\
\hline$\%$ caséine $\alpha_{\mathrm{s} 2}$ & 13,1 & 1,1 & 11,0 & 15,0 \\
\hline$\%$ caséine $\beta$ & 42,0 & 0,9 & 40,6 & 43,7 \\
\hline$\%$ caséine $\kappa$ & 10,4 & 0,7 & 8,9 & 11,4 \\
\hline
\end{tabular}

* Pour la variable MIC : $n=16$. Pour les proportions relatives des caséines : $n=13$ 


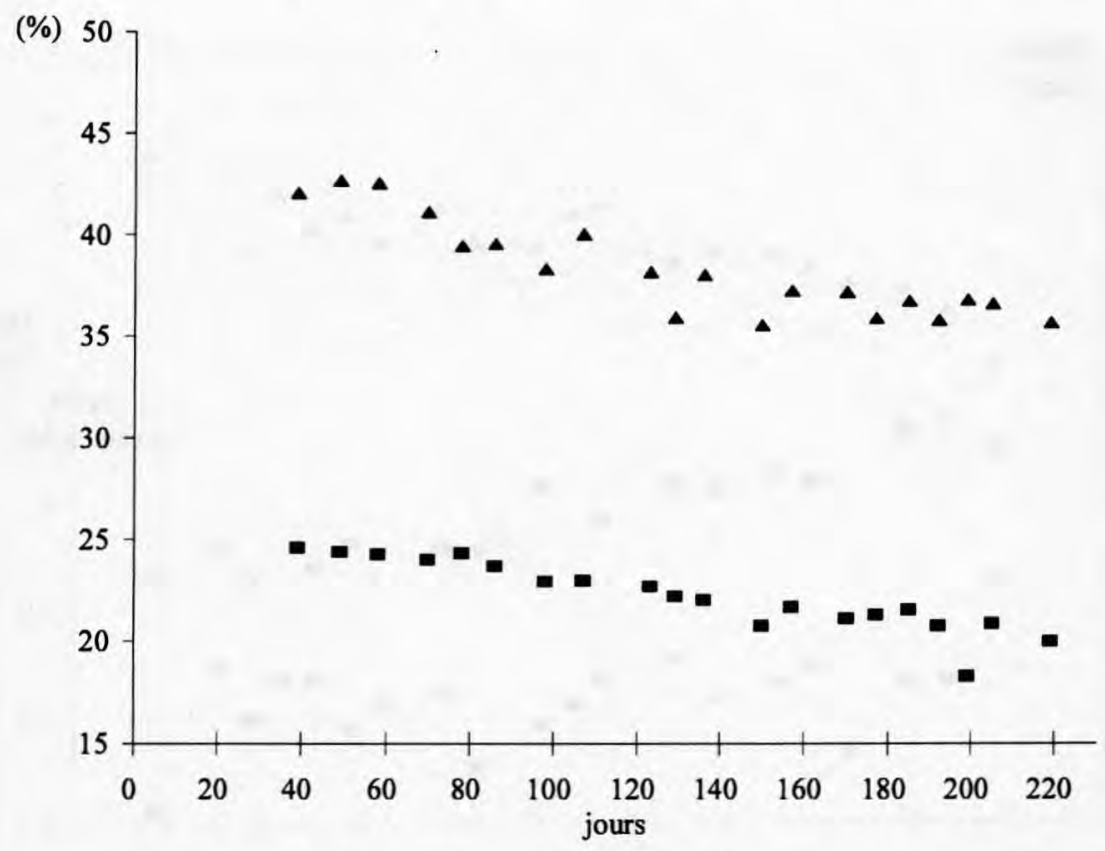

- Calcium sol. (Cas) \ Phosphore inorganique sol. (Pis)

Fig 4. Évolution des pourcentages de calcium (\% Cas) et phosphore inorganique solubles (\% Pis). Evolution of the percentages of diffusible calcium (\% Cas) and inorganic phosphorus (\% Pis).

de lactation, ne représente plus que $36 \%$ du total dans les derniers jours de collecte.

\section{Caractéristiques micellaires}

Le tableau II indique les valeurs moyennes, les écart types et les valeurs extrêmes observées pour les principales caractéristiques micellaires.

Le suivi des caractéristiques micellaires au cours de la période de production laitière met en évidence une augmentation significative de la taille des micelles, dont le diamètre passe de $175 \mathrm{~nm}$ à près de $200 \mathrm{~nm}$ durant les 120 premiers jours de collecte, puis se stabilise autour de cette valeur. Le degré d'hydratation des micelles ne pré- sente pas d'évolution marquée et reste pratiquement constant autour d'une valeur moyenne de $1,82 \mathrm{~g}$ eau/g matière sèche (MS), en excluant les 2 points extrêmes que l'on peut juger non représentatifs (fig 5).

La minéralisation calcique des micelles, estimée en effectuant le rapport entre la teneur en calcium colloïdal et la teneur en caséine, présente une décroissance régulière de 36 à 31 mg/g (fig 5).

En ce qui concerne les proportions relatives de caséines, elles restent pratiquement constantes au cours des 7 mois de production laitière, avec des pourcentages relatifs moyens des caséines $\alpha_{s 1}, \alpha_{s 2}, \beta$ et $\kappa$ respectivement égaux à $34,5,13,1,42,0$ et $10,4 \%$. Toutefois, au sein de la fraction $\alpha_{s}$, on peut relever une tendance à l'augmen- 


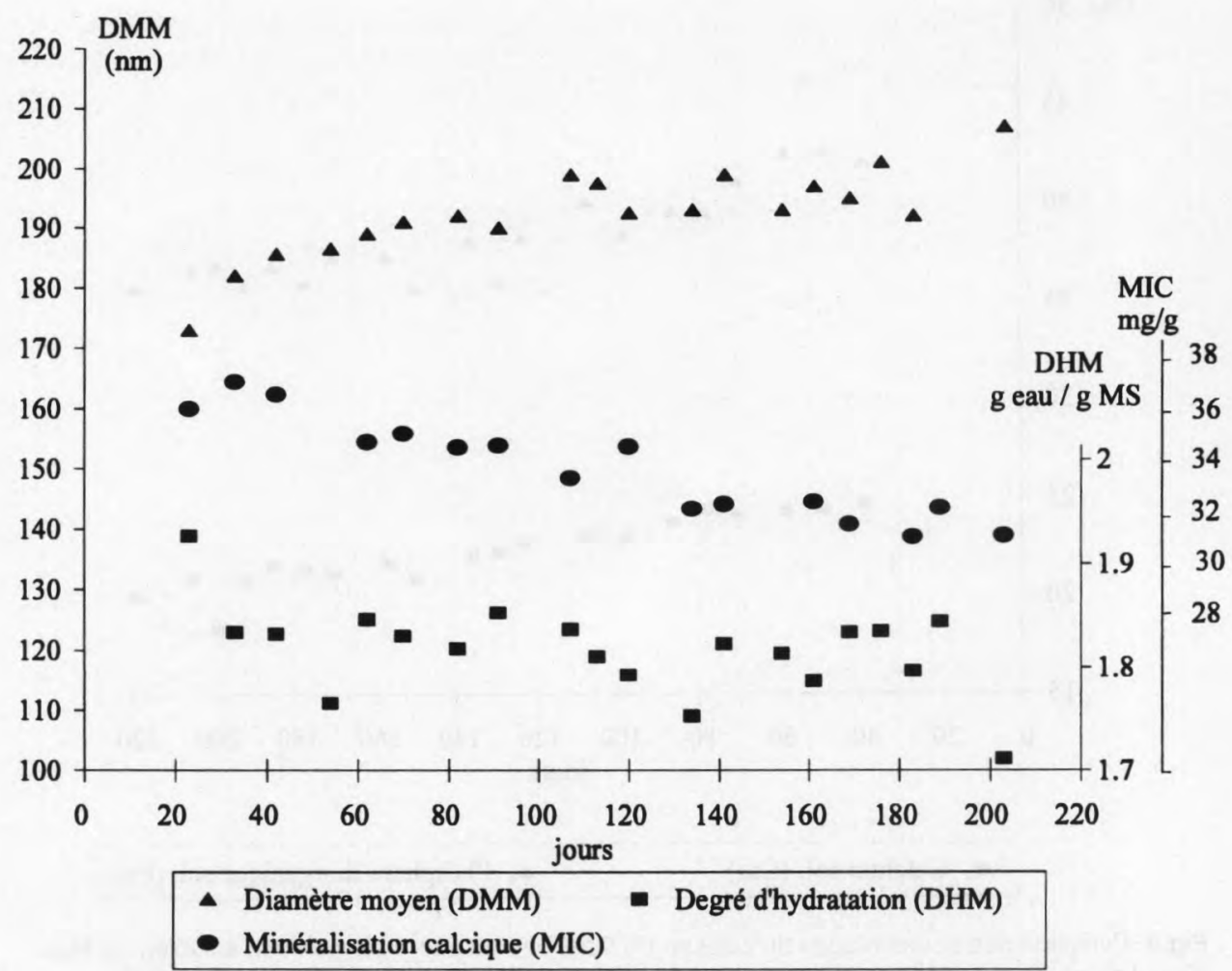

Fig 5. Évolution des caractéristiques micellaires : diamètre moyen (DMM), degré d'hydratation (DHM) et minéralisation calcique (MIC).

Evolution of micelle characteristics: Mean diameter (DMM), solvatation (DHM) and mineralization (MIC).

Tableau III. Paramètres de coagulation.

Rennet coagulation parameters.

\begin{tabular}{lcccc}
\hline & $\begin{array}{c}\text { Moyenne } \\
(\mathrm{n}=20)\end{array}$ & Écart type & \multicolumn{2}{c}{$\begin{array}{c}\text { Valeurs extrêmes } \\
\text { Min }\end{array}$} \\
& 19,5 & 2,11 & 16,8 & 23,8 \\
\hline $\begin{array}{l}\text { Temps de prise } \\
\text { Tp (min) }\end{array}$ & 5,26 & 0,68 & 4,21 & 6,49 \\
$\begin{array}{l}\text { Vitesse de raffermissement du gel } \\
\text { VRG (mm/min) }\end{array}$ & 59,8 & 1,5 & 56,3 & 61,7 \\
$\begin{array}{l}\text { Fermeté du gel } \\
\text { Ar (mm) }\end{array}$ & 15,2 & 2,15 & 11,5 & 18,5 \\
Sérum (p\%p) & & & & \\
\hline
\end{tabular}


tation de la proportion de caséine $\alpha_{\mathrm{s} 1}$, qui passe de 70 à près de $75 \%$ des caséines $\alpha_{\mathrm{s}}$ totales.

\section{Aptitude à la coagulation par la présure}

Le tableau III présente les valeurs moyennes et la variabilité des paramètres lactodynamiques du lait collecté par la fromagerie ayant fait l'objet d'un échantillonnage en 1992.

Les évolutions des différents paramètres de coagulation sont précisées sur les figures 6 et 7 . Le temps de prise augmente de manière régulière au cours de la période de production passant de 16,5 à 24 minutes. La vitesse de raffermissement suit une évo- lution inverse mais le paramètre caractérisant la fermeté du gel ( $\mathrm{Ar}$ ) ne présente pas d'évolution très marquée. Il croît légèrement durant la première partie de la période de production jusqu'au $140^{\circ}$ jour de collecte, puis ne varie plus beaucoup ensuite. L'aptitude du gel à l'égouttage diminue, quant à elle, de façon significative.

\section{DISCUSSION}

\section{Composition globale}

Concernant les principales caractéristiques du lait, les valeurs moyennes obtenues, ainsi que les évolutions observées en fonc-

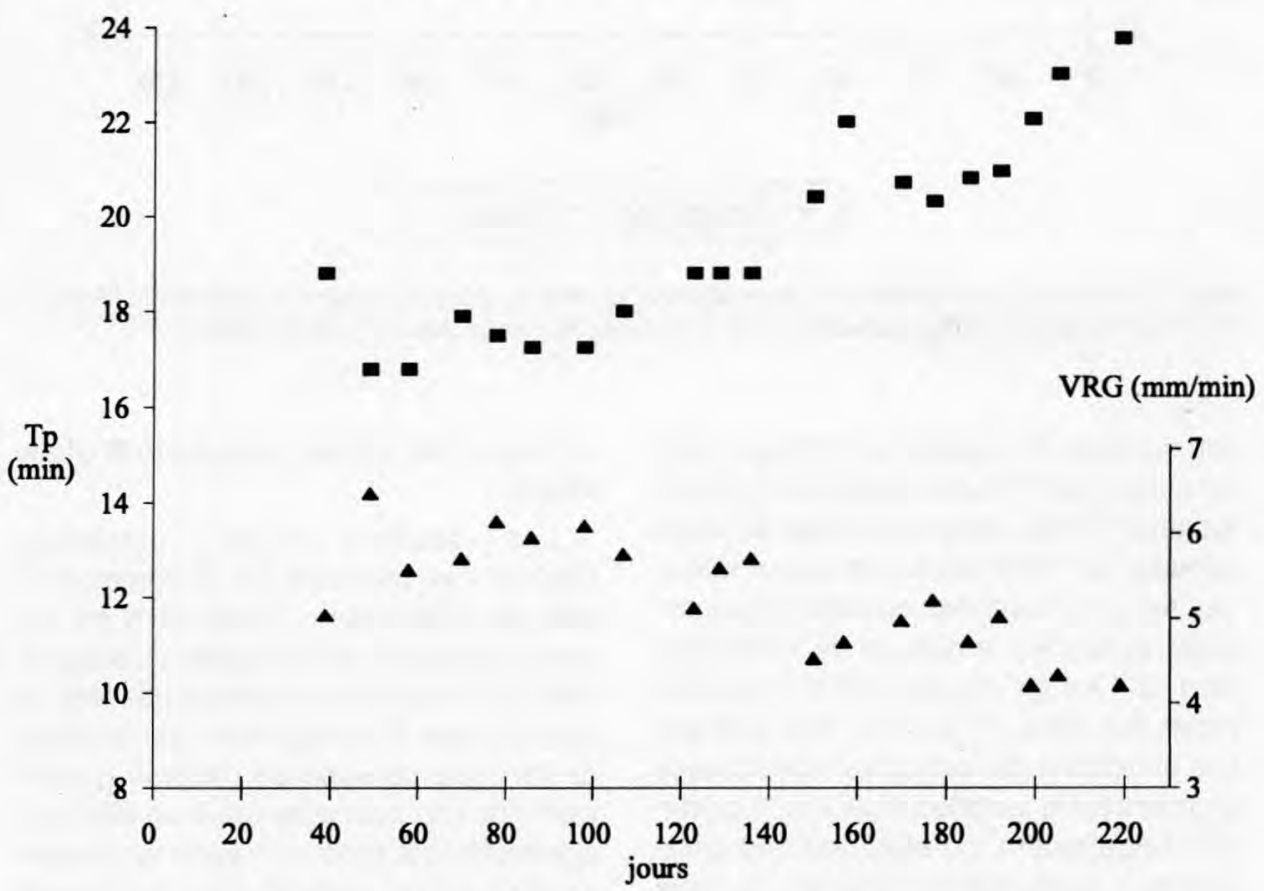

- Temps de prise (Tp) $\quad$ - Vitesse de raffermissement (VRG)

Fig 6. Évolution des paramètres de coagulation : temps de prise (Tp), vitesse de raffermissement du gel (VRG).

Evolution of rennet coagulation parameters: clotting time (Tp), gel firming rate (VRG). 


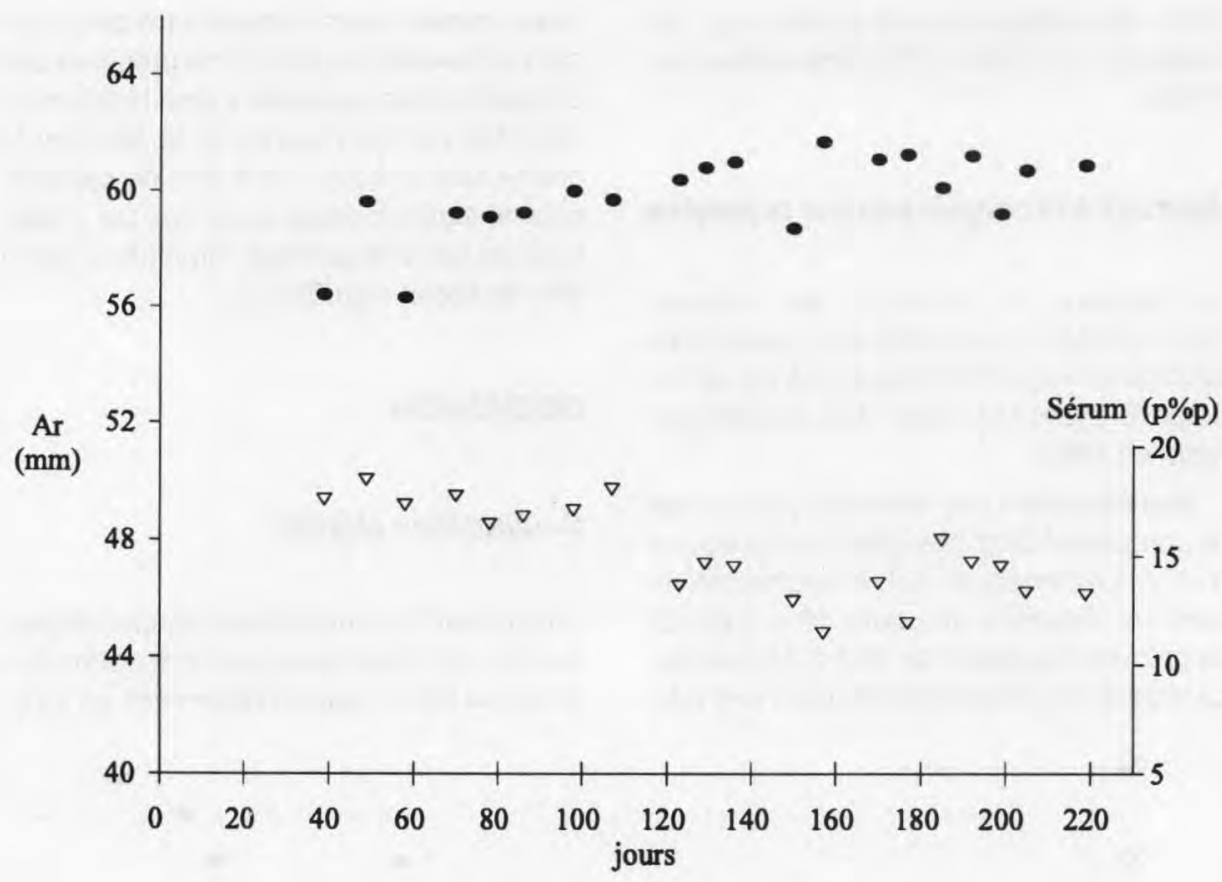

- Fermeté (Ar) $\nabla$ Sérum

Fig 7. Évolution des caractéristiques du coagulum: fermeté du gel (Ar), aptitude à l'égouttage (Sérum). Evolution of coagulum characteristics: curd firmness (Ar), whey draining ability (Sérum).

tion du stade de lactation sont très proches de celles décrites par Rivemale cité par Assenat (1985), dans une étude similaire conduite en 1982. Ainsi, cet auteur relève des teneurs moyennes en matière grasse, caséine, lactose et calcium de $74,3,46,8$, 48,1 et $1,94 \mathrm{~g} / \mathrm{l}$ respectivement, comparables aux nôtres $(74,0,44,1,46,6,1,89 \mathrm{~g} / \mathrm{l})$. Les évolutions des principaux constituants au cours de la lactation (figs 2 et 3 ) confirment également les observations antérieures : augmentation régulière du taux butyreux, diminution de la teneur en lactose, augmentation jusqu'au $160^{\mathrm{e}}$ jour puis stabilisation des concentrations en protéine et caséine, enfin courbes croissantes jusqu'au $140^{\circ}$ jour, puis décroissantes pour les teneurs en calcium, phosphore et citrate totaux.

Les résultats moyens présentés (tableau I) ne prennent pas en compte l'origine des échantillons analysés. II nous a paru intéressant de comparer statistiquement la composition moyenne des laits de chacune des 3 fromageries. Les résultats de l'analyse de variance (tableau I) montrent que l'on peut conclure à un effet non significatif de la zone de collecte sur la composition du lait, excepté pour les teneurs en lactose et phosphore $(P=0,03$ et $P=$ 0,02 respectivement). Bien que les valeurs du rapport caséine/protéine soient très proches pour les 3 fromageries, $82,5,82,4$, $82,2 \%$, l'effet de la zone de collecte sur 
cette variable apparaît faiblement significatif $(P=0,05)$. D'autre part, l'analyse statistique corrobore les évolutions de composition décrites en fonction de la période de production puisque l'effet «période» est très significatif pour toutes les variables étudiées (tableau I).

\section{Équilibres salins}

Les résultats relatifs aux équilibres salins présentent une bonne concordance avec les données publiées dans la littérature. Ainsi, O'Connor et Fox (1977), Polychroniadou et Vafopoulou (1986), et Lemoine (1990) déterminent des pourcentages de minéraux colloïdaux se situant entre 74,6 et $82 \%$ pour le calcium et entre 61,1 et $62,2 \%$ pour le phosphore inorganique. Il s'agit de valeurs proches de celles que nous proposons, respectivement 78 et $62 \%$. Holt et Jenness (1984) utilisant la technique d'ultrafiltration pour la séparation des phases, font état de pourcentages légèrement supérieurs, avec $90 \%$ de calcium et $70 \%$ de phosphore inorganique sous forme colloïdale. Ces différences de résultats sont probablement liées à la technique de séparation, comme l'ont montré Davies et White (1960).

Conformément au modèle micellaire proposé par Schmidt (1982), on distingue au sein de la fraction colloïdale deux formes de calcium. Une partie est liée directement aux groupements phosphoséryls des caséines, l'autre est présente sous forme de phosphate de calcium et assure la cohésion de la micelle. La nature chimique de ce phosphate de calcium étant encore discutée (eg Holt et al, 1989 ; Van Dijk, 1990), nous avons retenu l'hypothèse d'un phosphate de calcium colloïdal tricalcique sous forme amorphe (Schmidt, 1982) et calculé le coefficient de 1,935 qui correspond au rapport $\mathrm{Ca} / \mathrm{P}(\mathrm{p} / \mathrm{p})$ de ce composé de formule $\mathrm{Ca}_{9}\left(\mathrm{PO}_{4}\right)_{6}$. Le pourcentage de calcium lié directement aux caséines (\% CaN) est alors calculé grâce à l'équation suivante:

$\% \mathrm{CaN}=((\mathrm{Cac}-1,935 \times \mathrm{Pic}) / \mathrm{Cac}) \times 100$

où Cac: concentration en calcium colloïdal ; Pic: concentration en phosphore inorganique colloïdal.

Le pourcentage ainsi calculé est égal à $30 \%$. Les résultats de O'Connor et Fox (1977) et Lemoine (1990) sur lait de brebis permettent de calculer des valeurs de 35 et $29 \%$ respectivement, proches de notre estimation.

L'évolution des pourcentages de calcium et de phosphore inorganique solubles semble traduire un transfert progressif de constituants de la phase soluble vers la phase colloïdale au fur et à mesure de l'avancement de la période de production. On peut émettre l'hypothèse que l'augmentation de la teneur en caséine du lait, alors que les concentrations en calcium et phosphore inorganique restent relativement stables, entraîne un certain déficit minéral des micelles qui se traduit par un déplacement des équilibres salins au profit de la phase colloïdale. II s'agit d'un résultat nouveau et inattendu, car à notre connaissance, il n'est pas fait mention dans la littérature d'évolutions aussi nettes des équilibres salins durant la période de production laitière.

\section{Caractéristiques micellaires}

Les valeurs moyennes de degré d'hydratation, $1,82 \mathrm{~g}$ eau/g MS, et de diamètre moyen des micelles, $193 \mathrm{~nm}$, sont en accord avec les données existantes dans la littérature. Lemoine (1990), utilisant les mêmes méthodes de mesure, obtient des résultats similaires pour les laits de brebis de race Manech des Pyrénées-Atlantiques : $1,78 \mathrm{~g}$ eau/g MS pour l'hydratation des micelles et $205 \mathrm{~nm}$ pour le diamètre moyen. À titre de 
comparaison, les valeurs moyennes obtenues sur lait de chèvre (Remeuf et al, 1989) et lait de vache (Remeuf et al, 1991) sont respectivement de $255 \mathrm{~nm}$ et $175 \mathrm{~nm}$ pour le diamètre et de 1,75 et $2,10 \mathrm{~g}$ eau/g MS pour le degré d'hydratation.

L'évolution nette et significative des dimensions micellaires dans le lait de brebis, au cours de la lactation, est un résultat nouveau. On peut se demander si l'augmentation de la taille des micelles n'est pas liée à l'accroissement de la richesse du lait en matière protéique. Toutefois, des travaux réalisés sur lait de vache ne semblent pas aller dans ce sens : selon Rose et Colvin (1966) et Remeuf et al (1991), le diamètre des micelles n'est pas corrélé à la concentration en caséine ou en protéine. Remeuf et al (1989) notent même, sur lait de chèvre, une corrélation négative entre ces 2 variables.

Quant au degré de minéralisation calcique, la valeur moyenne calculée, de 33,4 $\mathrm{mg}$ de $\mathrm{Ca} / \mathrm{g}$ de caséine, est en total accord avec les résultats de Holt et Jenness (1984), Polychroniadou et Vafopoulou (1986) et Lemoine (1990). Le lait de brebis présente une minéralisation calcique proche de celles observées dans les laits bovin et caprin. Ainsi, Holt et Jenness (1984) et Remeuf et al $(1989$; 1991) relèvent des valeurs respectivement égales à 31 et $30 \mathrm{mg} / \mathrm{g}$ pour le lait de vache et 32 et $36 \mathrm{mg} / \mathrm{g}$ pour le lait de chèvre. La minéralisation calcique diminue sensiblement au cours de la période de production laitière et cette observation rejoint celle faite au sujet du déplacement des équilibres salins vers la phase colloïdale. Finalement, on peut supposer qu'il se produit avec l'avancement de la lactation une évolution des conditions de la synthèse du lait qui entraîne une modification de certaines caractéristiques physico-chimiques telles que la taille des micelles et leur degré de minéralisation. II s'agit d'une hypothèse plausible pour expliquer les évolutions observées, mais les mécanismes physico- chimiques et/ou physiologiques mis en jeu demeurent largement incompris.

Les données concernant les proportions relatives des caséines montrent que les caséines $\alpha_{s}$ constituent la fraction prépondérante, avec $47,6 \%$ du total des caséines, devant la caséine $\beta(42 \%)$, la caséine $\kappa$ représentant $10,4 \%$. Nos résultats sont assez proches de ceux obtenus par quelques autres auteurs (Juarez et al, 1984 ; Anifantakis, 1986 ; Ono et al, 1989). En revanche, Brochet (1982), Law et al (1992) et Muir et al (1993) notent, quant à eux, une proportion de caséine $\beta$ un peu supérieure à celle des caséines $\alpha_{\mathrm{s}}$. À notre connaissance, aucun article ne fait mention de l'utilisation de la technique HPLC en phase inverse pour la séparation et la quantification des caséines ovines. Les pourcentages relatifs de chacune de ces fractions évoluent peu au cours de la période de production. Tanev et Youcheva (1976) relèvent, quant à eux, une augmentation du pourcentage de caséine $\kappa$ en fin de lactation. En revanche, Brochet (1982) ainsi que Muir et al (1993) n'observent pas d'évolution des proportions des caséines $\alpha_{s}$, $\beta$ et $\kappa$. Un certain nombre d'études effectuées sur lait de vache par différents auteurs (eg Davies et Law, 1980 ; Kroeker et al, 1985) tendent, de même, à montrer l'absence de variations saisonnières systématiques des proportions relatives des caséines.

\section{Aptitude à la coagulation par la présure}

En ce qui concerne l'aptitude du lait à la coagulation par la présure, il est difficile de comparer nos résultats avec ceux obtenus par d'autres équipes, car les conditions opératoires (température, dose de présure) ne sont pas nécessairement identiques.

L'évolution croissante du temps de prise au cours de la période de production (fig 6) confirme les données de la littérature. Des travaux antérieurs ont également mis en 
évidence un allongement du temps de coagulation du lait de brebis avec le stade de lactation (Ubertalle et al, 1990 ; DelacroixBuchet et al, 1994), indépendamment de l'influence du $\mathrm{pH}$. Nos résultats ne permettent pas non plus de relier l'accroissement du temps de prise avec une évolution inverse du $\mathrm{pH}$. On peut émettre l'hypothèse que les modifications des équilibres salins et des caractéristiques micellaires au cours de la période de production sont en partie à l'origine de cette évolution du temps de prise. En particulier, le phosphate de calcium colloïdal joue un rôle essentiel dans la formation du coagulum présure (Zittle, 1970 ; Shalabi et Fox, 1982) et la réduction de la minéralisation calcique est un facteur susceptible d'expliquer, en partie, l'augmentation du temps de coagulation observée. Ainsi, Remeuf et al (1991) ont noté, sur lait de vache, une corrélation négative significative entre ces 2 variables. Mais l'allongement du temps de prise pourrait être lié aussi à l'accroissement des dimensions micellaires (eg Ekstrand et al, 1980 ; Niki et Arima, 1984 ; Ford et Grandison, 1986).

La diminution de la vitesse de raffermissement au cours de la période de production (fig 6) est en accord avec les résultats de Delacroix-Buchet et al (1994). En outre, elle confirme l'existence d'une corrélation négative entre vitesse de raffermissement et temps de prise, signalée par les mêmes auteurs.

La fermeté du gel mesurée ici à l'aide du paramètre Ar augmente faiblement avec l'avancement de la lactation (fig 7). Delacroix-Buchet et al (1994) ne notent pas d'évolution régulière du paramètre Ar alors que Ubertalle et al (1990), évaluant la fermeté par la variable $\mathrm{A} 30$ (fermeté du gel 30 min après l'emprésurage), observent une diminution de cette variable durant la période de production. Ces résultats paraissent un peu surprenants compte tenu de la relation bien établie entre la fermeté du gel présure et la richesse du lait en caséine (Lenoir et Schneid, 1987). Les évolutions décrites paraissent en effet assez faibles, voire contradictoires au regard de l'enrichissement du lait en caséine au cours de la période de lactation. Le paramètre $\mathrm{A} 30$ n'est probablement pas satisfaisant pour décrire la fermeté du coagulum présure du lait de brebis car il dépend étroitement du temps de prise. En ce qui concerne les évolutions du paramètre $\mathrm{Ar}$, il est probable que l'augmentation de la teneur en protéine soit contrebalancée par des effets négatifs dus à la modification des équilibres salins ou des caractéristiques micellaires. En effet, la corrélation positive établie dans le lait de vache entre minéralisation phospho-calcique et fermeté du gel (Remeuf et al, 1991), et celle, négative, entre la fermeté et le diamètre des micelles (Niki et Arima, 1984; Ford et Grandison, 1986 ; Delacroix-Buchet et al, 1993), constituent des explications possibles de cette évolution peu marquée du paramètre Ar.

II n'existe pas à notre connaissance de travaux ayant décrit l'évolution de l'aptitude à l'égouttage du lait de brebis durant la lactation. L'évolution de la variable "Sérum" (fig 7) montre que la capacité du gel à l'exsudation de lactosérum diminue au cours de la lactation, corrélativement à l'augmentation de la richesse du lait en matière grasse et matière protéique. Ce résultat est en accord avec les observations faites par Maubois et Mocquot (1971) qui montrent que les gels de laits enrichis en caséine par la technique d'ultrafiltration s'égouttent peu. De même, Storry et al (1983) mettent en évidence une moins bonne aptitude à l'égouttage des laits avec l'augmentation de la teneur en matière grasse. Enfin, le lait de brebis étant beaucoup plus riche en matière sèche que les laits bovin et caprin, il en résulte que le volume de sérum exsudé, dans des conditions déterminées et pour une quantité de lait donnée, est plus faible que pour le lait des 2 autres espèces (Storry et al, 1983). 
II est intéressant de constater que les évolutions relevées en ce qui concerne les paramètres de coagulation enzymatique dans le lait de brebis, sont proches de celles mises en évidence par différents auteurs dans le lait de vache. Ainsi, plusieurs études ont décrit une augmentation assez nette du temps de prise du lait bovin au cours de la période de lactation (Mariani et al, 1982 ; Okigbo et al, 1985 ; Davoli et al, 1987 ; Rampilli et al, 1988). La vitesse de raffermissement du gel varie en sens inverse (Davoli et al, 1987 ; Rampilli et al, 1988). Quant à la fermeté du gel mesurée par le paramètre A30 (Mariani et al, 1982 ; Okigbo et al, 1985 ; Davoli et al, 1987) elle suit une évolution proche de celle décrite pour le lait de brebis par Ubertalle et al (1990).

\section{CONCLUSION}

Ce travail a permis de suivre l'évolution des caractéristiques physico-chimiques et des paramètres de coagulation du lait de brebis dans la région de Roquefort. II confirme notamment l'augmentation significative des teneurs en matière grasse et matière protéique au cours de la période de production laitière. La comparaison de laits provenant de 3 zones de collecte n'a révélé pratiquement aucune différence significative de composition entre les 3 types de lait. Ce travail a mis en évidence des résultats originaux, en ce qui concerne notamment les équilibres salins et les caractéristiques micellaires. II serait intéressant maintenant d'établir des corrélations entre ces différentes variables, et en particulier, celles qui lient les caractéristiques physico-chimiques et les paramètres de coagulation. Des données relatives à des laits individuels ou de petit mélange conviennent parfaitement à cette approche, du fait de leur grande variabilité. Un travail de ce type est en cours actuellement dans nos laboratoires et devrait faire l'objet prochainement de publications.

\section{REMERCIEMENTS}

Ce travail a été réalisé avec l'assistance technique de MT Le Tilly, ainsi que l'équipe du laboratoire de physico-chimie de la Société des caves de Roquefort, Nous les en remercions.

Nous remercions également $\mathrm{F}$ Barillet (INRASAGA Toulouse) pour sa lecture critique du manuscrit.

\section{REFERENCES}

Anifantakis EM (1986) Comparison of the physico-chemical properties of ewes' and cows' milk. Int Dairy Fed Bull 202, 42-53

Assenat $L$ (1985) Le lait de brebis. Composition et propriétés. In : Lait et produits laitiers. I. Les laits de la mamelle à la laiterie (Luquet FM, ed). Lavoisier, Tec \& Doc, Paris

Brochet M (1982) Étude des laits de brebis. Méthodes de dosage des principaux constituants et composition des protéines. Thèse $3^{\circ}$ cycle. Université ClaudeBernard, Lyon I

Davies DT, Law AJR (1980) The content and composition of protein in creamery milks in south-west Scotland. J Dairy Res 47, 83-90

Davies DT, White JCD (1960) The use of ultrafiltration and dialysis in isolating the aqueous phase of milk and in determining the partition of milk constituents between the aqueous and disperse phases. J Dairy Res 27, 171-190

Davoli A, Dall'Olio S, Russo V (1987) Ricerche sul controllo funzionale delle caratteristiche lattodinamometriche nelle bovine da latte. (Milk test records of the lactodynamographic properties in dairy cattle). Sci Tec Latt-Casearia 38, 547-558

Delacroix-Buchet A, Lefier D, Nuyts-Petit V (1993) Polymorphisme de la caséine $\kappa$ de trois races bovines françaises et aptitude à la coagulation. Lait 73, 61-72

Delacroix-Buchet A, Barillet F, Lagriffoul G (1994) Caractérisation de l'aptitude fromagère des laits de brebis Lacaune à l'aide d'un Formagraph. Lait 74, 173-186

Duranti E, Casoli C (1991) Variazione della composizione azotata e dei parametri lattodinamografici di latte di pecora in funzione del contenuto di cellule somatiche. (Variations in the nitrogen composition and in the lactodynamographic parameters of ewe's milk in relation to somatic cell content). Zootec Nutr Anim 17, 99-105

Ekstrand B, Larsson-Raznikiewicz M, Perlmann C (1980) Casein micelle size and composition related to the enzymatic coagulation process. Biochim Biophys Acta $630,361-366$ 
FIL (Fédération internationale de laiterie) (1967) Détermination de la teneur en phosphore du lait. Norme 42

Ford GD, Grandison AS (1986) Effect of size of casein micelles on coagulation properties of skim milk. J Dairy Res 53, 129-133

Holt C, Jenness R (1984) Interrelationships of constituents and partition of salts in milk samples from eight species. Comp Biochem Physiol 77 A, 275282

Holt C, Vankemenade MJJM, Nelson LSJ, Sawyer L, Harries JE, Bailey RT, Hukins DWL (1989) Composition and structure of micellar calcium phosphate. J Dairy Res 56, 411-416

Jaubert A, Martin P (1992) Reverse-phase HPLC analysis of goat caseins. Identification of $\alpha_{\mathrm{s} 1}$ and $\alpha_{\mathrm{s} 2}$ genetic variants. Lait 72, 265-247

Journal Officiel de la République Française, 20 mars 1981, NC 2908

Juarez M, Ramos M, Goicoechea A, Jimenez-Perez S (1984) Main components, nitrogen fractions and mineral elements of Manchega ewe's milk. Chem Mikrobiol Technol Lebensm 8, 143-146

Kroeker EM, Ng-Kwai-Hang KF, Hayes JF, Moxley JE (1985) Effects of environmental factors and milk protein polymorphism on composition of casein fraction in bovine milk. J Dairy Sci 68, 1752-1757

Law AJR, Papoff CM, Dalgleish DG, Campus RL (1992) Quantitative fractionation of ovine casein by cationexchange FPLC. Milchwissenschaft 47, 279-282

Lemoine $\mathrm{R}$ (1990) Composition et rendement fromager du lait de brebis en Pyrénées-Atlantiques. Compte rendu de fin d'étude ITOVIC et SICA-CREOM ; édn ITOVIC, Paris

Lenoir J, Schneid N (1987) L'aptitude du lait á la coagulation par la présure. In : Le fromage, $2^{\circ}$ ed (Eck $A$, ed). Lavoisier, Tec \& Doc, Paris

Manfredini M, Cavani C, Chiarini R. Sanguinetti V, Zarri MC (1987) Effeti dell'insilato di mais sulle caratteristiche qualitative del latte e del formaggio di pecora. (Effects of maize silage on the qualitative characteristics of ewe milk and cheese). Zootec Nutr Anim $13,21-28$

Manfredini M, Tassinari M, Zarri MC (1992) Caratteristiche chimico-fisiche, contenuto in cellule somatiche ed attitudine alla coagulazione di latte individuale di pecore allevate in Emilia-Romagna. (Physico-chemical characteristics and coagulation properties of milk from individual ewes). Sci Tec Latt-Casearia 43, 113-125

Mariani P. Pecorari M, Fossa E (1982) Lè caratteristiche di coagulazione del latte in rapporto allo stadio della lattazione ed ai livelli di produzione. (Rennet coagulation of milk in relation to lactation stage and yield levels of cows). Sci Tec Latt-Casearia 33, 409425
Marshall RJ (1982) An improved method for measurement of the syneresis of curd formed by rennet action on milk. J Dairy Res 49, 329-336

Maubois JL, Mocquot G (1971) Préparation de fromage à partir de "pré-fromage liquide" obtenu par ultrafiltration du lait. Lait 51, 495-533

Muir DD, Horne DS, Law AJR, Steele W (1993) Ovine milk.1. Seasonal changes in composition of milk from commercial Scottish flock. Milchwissenchaft $48,363-366$

Niki R, Arima S (1984) Effects of size of casein micelle on firmness of rennet curd. Jpn J Zootech Sci 55 , $409-415$

Nuñez M, Medina M, Gaya P (1989) Ewes' milk cheese: technology, microbiology and chemistry. J Dairy Res $56,303-321$

O'Connor P, Fox PF (1977) The proteins and salts of some non-bovine milks. J Dairy Res 44, 607-609

Okigbo LM, Richardson GH, Brown RJ, Ernstrom CA (1985) Variation in coagulation properties of milk from individual cows. J Dairy Sci 68, 822-828

Ono $T$, Kohno $H$, Odagiri S, Takagi $T$ (1989) Subunit components of casein micelles from bovine, ovine, caprine and equine milks. J Dairy Res 56, 61-68

Polychroniadou A, Vafopoulou A (1985) Variations of major mineral constituents of ewe milk during lactation. J Dairy Sci $68,147-150$

Polychroniadou A, Vafopoulou A (1986) Salt distribution between the colloidal and soluble phases of ewes' milk. J Dairy Res 53, 353-358

Ramos M, Juarez M (1981) The composition of ewe's and goat's milk. Int Dairy Fed Bull 140, 5-19

Rampilli M, Caroli A, Bolla P, Pirlo G (1988) Relazion tra genotipi lattoproteici composizione caseinica e attitudine alla coagulazione del latte nel corso della lattazione. (Relationships among milk proteins genotypes casein composition and clotting aptitude of milk during lactation). Sci Tec Latt-Casearia 39, 262-279

Remeuf F, Lenoir J, Duby C (1989) Étude des relations entre les caractéristiques physico-chimiques des laits de chèvre et leur aptitude à la coagulation par la présure. Lait 69, 499-518

Remeuf F, Cossin V, Dervin C, Lenoir J, Tomassone R (1991) Relations entre les caractéristiques physicochimiques des laits et leur aptitude fromagère. Lait $71,397-421$

Richardson BC, Creamer LK, Pearce KN, Munford RE (1974) Comparative micelle structure. II. Structure and composition of casein micelles in ovine and caprine milk as compared with those in bovine milk. J Dairy Res 41, 239-247

Rose D, Colvin JR (1966) Appearance and size of micelles from bovine milk. J Dairy Sci 49, 1091-1097

Rowland SJ (1938) The determination of the nitrogen distribution in milk. J Dairy Res 9, 42-46

Saleem RM, Mahmoud WA, Younis YA (1986) Nitrogen distribution, molecular weight and size of casein 
micelles in Awassi sheep's milk. Egypt J Dairy Sci 14, 81-86

Schmidt DG (1982) Association of caseins and casein micelle structure. In : Developments in Dairy Chemistry. I. Proteins (Fox PF, ed). Applied Science Publishers, Barking

Shalabi SI, Fox PF (1982) Influence of pH on the rennet coagulation of milk. J Dairy Res 49, 153-157

Storry JE, Grandison AS, Millard D, Owen AJ, Ford GD (1983) Chemical composition and coagulating properties of renneted milks from different breeds and species of ruminant. J Dairy Res $50,215-229$

Tanev G, Youcheva G (1976) Comparative study of milk protein of East Friesian and Zlatushki ewes. II. Changes in the percentage ratios of protein fractions during the milking period. Anim Sci 13, (7), 27-31
Ubertalle A, Ambrosoli R, Errante J (1990) Tipizzazione lattodinamografica del latte ovino. Sci Tec LattCasearia 41, 53-63

Van Dijk HJM (1990) The properties of casein micelles.1. The nature of the micellar calcium phosphate. Neth Milk Dairy J 44, 65-81

Voutsinas LP, Delegiannis C, Katsiari MC, Pappas C (1988) Chemical composition of Boutsiko ewe milk during lactation. Milchwissenschaft 43, 766-771

Zannoni M, Annibaldi S (1981) Standardization of the renneting ability of milk by Formagraph. I. Sci Tec Latt-Casearia, 32, 79-94

Zittle CA (1970) Influence of phosphate and other factors on the rennin gel obtained with whole casein and with $\mathrm{\kappa}$-casein in the presence of calcium salts. J Dairy Sci 53, 1013-1017 